\title{
Quantitative and qualitative comparison of morphometric outcomes after endoscopic and conventional correction of sagittal and metopic craniosynostosis versus control groups
}

\author{
Matthias Schulz, Dr med,, Linda Liebe-Püschel,, Karl Seelbach, ${ }^{1}$ Laura Paulikat, ${ }^{1}$ \\ Felix Fehlhaber, Dipl-Ing, ${ }^{2}$ Karin Schwarz, Dr med, ${ }^{1}$ Christoph Blecher, Dr med, ${ }^{3}$ and \\ Ulrich-Wilhelm Thomale, Dr med ${ }^{1}$ \\ ${ }^{1}$ Pediatric Neurosurgery, Charité Universitätsmedizin Berlin; ${ }^{2}$ Fraunhofer Institute for Production Systems and Design Technology \\ (IPK); and ${ }^{3}$ Cranioform, Berlin, Germany
}

\begin{abstract}
OBJECTIVE Surgical correction for sagittal and metopic craniosynostosis (SCS and MCS) aims to alter the abnormal cranial shape to resemble that of the normal population. The achieved correction can be assessed by morphometric parameters. The purpose of the presented study was to compare craniometric parameters of control groups to those same parameters after endoscopic and conventional (open) correction.

METHODS The authors identified 4 groups of children undergoing surgical treatment for either SCS or MCS, with either endoscopic (SCS, $n=17$; MCS, $n=16$ ) or conventional (SCS, $n=29$; MCS, $n=18$ ) correction. In addition, normal control groups of nonaffected children who were $6(n=30)$ and $24(n=18)$ months old were evaluated. For all groups, several craniometric indices calculated from 3D photographs were compared for quantitative analysis. For qualitative comparison, averages of all 3D photographs were generated for all groups and superimposed to visualize relative changes.

RESULTS For children with SCS, the cephalic index and coronal circumference index significantly differed preoperatively from those of the 6 -month normal controls. The respective postoperative values were similar to those of the 24-month normal controls after both endoscopic and conventional correction. Similarly, for children with MCS, indices for circumference and diagonal dimension that were significantly different preoperatively became nonsignificantly different from those of 24-month normal controls after both endoscopic and conventional correction. The qualitative evaluation of superimposed average 3D head shapes confirmed changes toward normal controls after both treatment modalities for SCS and MCS. However, in SCS, the volume gain, especially in the biparietal area, was more noticeable after endoscopic correction, while in MCS, relative volume gain of the bilateral forehead was more pronounced after conventional correction. The average 3D head shapes matched more homogeneously with the average of normal controls after endoscopic correction for SCS and after conventional correction for MCS.

CONCLUSIONS This quantitative analysis confirms that the performed surgical techniques of endoscopic and conventional correction of SCS and MCS alter the head shape toward those of normal controls. However, in a qualitative evaluation, the average head shape after endoscopic technique for SCS and conventional correction for MCS appears to be closer to that of normal controls than after the alternative technique. This study reports on morphometric outcomes after craniosynostosis correction. Only an assessment of the whole multiplicity of outcome parameters based on multicenter data acquisition will allow conclusions of superiority of one surgical technique.
\end{abstract}

https://thejns.org/doi/abs/10.3171/2021.1.FOCUS20988

KEYWORDS sagittal; metopic craniosynostosis; endoscopic; conventional correction

ABBREVIATIONS $\mathrm{CCI}=$ coronal circumference index; $\mathrm{CFI}=$ circumference index; $\mathrm{Cl}=$ cephalic index; $\mathrm{DI}=$ diagonals index; $\mathrm{ECI}=$ exocanthion index; $\mathrm{FOR}=$ frontoorbital remodeling; MCS = metopic craniosynostosis; NFA = nasofrontal angle; SCS = sagittal craniosynostosis; $\mathrm{VHI}=$ vertex height index.

SUBMITTED November 19, 2020. ACCEPTED January 19, 2021.

INCLUDE WHEN CITING DOI: 10.3171/2021.1.FOCUS20988. 
A LTHOUGH craniosynostosis is a rare condition with a prevalence of approximately 7.2 cases per 10,000 live births, ${ }^{1}$ it is commonly seen in pediatric neurosurgical practice. Indication for surgical correction is based on the severity of the typically associated morphological phenotype and is usually performed in infancy. In addition to conventional open remodeling techniques, endoscopically assisted strip craniectomy followed by helmet therapy has gained popularity. ${ }^{2-5}$

While several reports compare the efficacy and safety of endoscopic procedures to conventional open procedures, the literature comparing morphometric data after different types of correction (besides cephalic index [CI] for sagittal craniosynostosis [SCS]) remains scarce. ${ }^{6-9}$ The same is true for comparison of morphometric data with a sample of the age-matched nonaffected population. ${ }^{10-12}$ Therefore, an evaluation of morphometric outcomes of children with the two most common types of single-suture craniosynostosis (SCS and metopic craniosynostosis [MCS]) was performed. For this retrospective study, several craniometric parameters of children with SCS and MCS, and of unaffected children serving as normal controls, were evaluated to quantify the development of head shapes after correction. Averages of pre- and postoperative 3D head shapes were compared to controls for qualitative evaluation.

\section{Methods}

\section{Clinical Management}

Ethical approval of the study design by the institutional ethics committee was confirmed prior to conducting the study. All children were routinely followed at the craniofacial outpatient clinic at Charité Universitätsmedizin Berlin with serial clinical appointments as well as by documentation using 3D photography (Vectra M5-360 Head System, Canfield Scientific). Parents were counseled about possible options for surgical correction according to the child's age. Before the age of 3 months, both endoscopic and conventional correction were discussed. The type of surgical correction was chosen according to the parents' preference. For children older than 3 months, only the conventional surgical technique was offered.

\section{Conventional Correction}

Surgical correction for SCS and MCS was performed in standard fashion. The correction of SCS was preferentially performed during the 6th month of life by a biparietal craniectomy $11 \mathrm{~cm}$ wide in diameter, including the fused suture together with radial barrel-stave incisions. Outward bending of the circumferential bone segments immediately increased the biparietal diameter. Parents were encouraged to place the child on the back of his or her head for the following weeks.

MCS correction was preferentially performed at the age of 9-11 months by frontoorbital remodeling (FOR). After bifrontal craniotomy and excision of the bandeau, remodeling of the forehead and reshaping of the bandeau were performed to increase the width of the forehead. The remodeled bandeau was secured by resorbable plates (KLS Martin) on the inside and, together with the forehead, fixed in an advanced position.

\section{Endoscopically Assisted Strip Craniectomy}

The age at which endoscopically assisted strip craniectomy was performed was approximately 3 months. The removal of a bone strip along the fused suture was performed via one (SCS) or two (MCS) small skin incisions. With endoscopic assistance, the bone was dissected from the dura mater prior to resection. For MCS, the craniectomy segment of 2- to 3-cm width was resected between the anterior fontanelle and the nasion. For SCS, a 2- to 3-cmwide strip was excised between the anterior fontanelle and lambda. After recession of the perioperative swelling, an individually manufactured cranial orthosis (Cranioform) was fitted. The duration of helmet therapy was individually determined depending on the achieved correction and underlying craniosynostosis type.

\section{Control and Patient Cohorts}

Three-dimensional photography was used to document all head shapes. Two cohorts of children at 6 and 24 months of age with normal head shapes as judged by parents, a clinical examination, and individual history were recruited as normal controls. The latter groups were recruited by advertisements in local kindergartens, at preventive medical checkups in pediatric practices, and from the children of hospital staff, and included only maturely born children without conditions known to affect cranial shape and without history of trauma or previous cranial surgery. For the review of the results after craniosynostosis correction, only children with $3 \mathrm{D}$ photographs documenting the preoperative state and postoperative followup (at a preferred age of $24 \pm 6$ months) were considered.

\section{Quantitative Analysis}

For the quantitative analysis of 3D photographs, Cranioform Analytics software (version 4.0) was used to retrieve craniometric data. After marking landmarks (nasion, tragus, superior otobasion, lateral canthus [exocanthion], subnasion, and pogonion) on the 3D photograph, the software computes an " $\mathrm{x}-\mathrm{y}-\mathrm{z}$ " coordinate system and several axial planes through the neurocranium parallel to a plane across the skull base (defined by the tragions and the exocanthions). For each plane and for the whole 3D data set, metric dimensions are calculated by this software (volumes, angles, distances, and circumferences). To allow comparison of metric parameters in axial dimensions, the plane with the largest circumference (above the auricle) was consistently used. To minimize influence of physiological head growth, only relations (indices) but no absolute measurements were compared.

For SCS, the following indices were evaluated: CI (length/width), coronal circumference index (CCI; coronal circumference/cranial base width), and vertex height index (VHI; vertex height/cranial base width). For MCS, the following indices were evaluated: circumference index (CFI; circumference/[length + width]), $30^{\circ}$ diagonals in$\operatorname{dex}\left(\mathrm{DI}\right.$; [left + right $30^{\circ}$ diagonal $] /[2 \times$ length $]$ ), and exocanthion index (ECI; midpoint to left + right exocanthion distance/[2 $\times$ anterior part of the length]). Note that for the ECI, the distance "midpoint to left + right exocanthion" is measured at the level of the skull base, whereas "anterior 
TABLE 1. Patient characteristics

\begin{tabular}{|c|c|c|c|c|c|c|}
\hline \multirow[b]{2}{*}{ Characteristic } & \multicolumn{2}{|c|}{ Normal Controls } & \multicolumn{2}{|c|}{ SCS } & \multicolumn{2}{|c|}{ MCS } \\
\hline & 6 Mos & 24 Mos & Endoscopic & Conventional & Endoscopic & Conventional \\
\hline No. of patients & 30 & 18 & 17 & 29 & 16 & 18 \\
\hline Females/males & $14 / 16$ & $9 / 9$ & $5 / 12$ & $2 / 27$ & $5 / 11$ & $6 / 12$ \\
\hline \multicolumn{7}{|l|}{ Age, mos $^{*}$} \\
\hline At scan for normal controls & $6.2(5.1-7.6)$ & $25(21.9-26.4)$ & & & & \\
\hline At preop scan & & & $2.9(2.1-3.7)$ & $5.5(1.5-9.1)$ & $2.7(1.4-4.3)$ & $8.9(2.9-18.9)$ \\
\hline At operation & & & $3.0(2.1-3.9)$ & $6.1(4.8-9.7)$ & $3.0(2.0-4.4)$ & $9.7(8.4-19.2)$ \\
\hline At postop scan & & & $24.6(17.5-33.6)$ & $20.1(18.1-27.2)$ & $23.8(17.7-33.5)$ & $21.4(18.2-25.0)$ \\
\hline $\begin{array}{l}\text { Duration of helmet therapy if } \\
\text { applicable, mos }{ }^{*}\end{array}$ & & & $5.0(2.9-14.5)$ & & $14.5(7.5-18.9)$ & \\
\hline
\end{tabular}

* Data are given as the number of patients or the median (range).

part of the length" describes the anterior part of the anteroposterior diameter from the midpoint to the forehead, measured at the cross-section with the largest circumference above the ear. In addition, the nasofrontal angle (NFA) was recorded (back of the nose/glabella) for both. The same measurements were assessed for the children in the control groups.

\section{Qualitative Analysis}

To allow a qualitative analysis of the head shapes and comparison with normal controls, an average 3D head surface model of all individuals of each group was calculated using specifically developed software (MeshMerging, Fraunhofer Institute for Production Systems and Design Technology)..$^{13}$

Briefly, to calculate an average 3D head shape of all point clouds, the point clouds are registered to each other by aligning tilt, pitch, and rolling of the whole point cloud to best fit nasion and bilateral tragions. All point clouds are separated into a cranial and facial area; the facial area is subsequently excluded from averaging. Corresponding points of each cloud are then found by a point-to-normal method originated at the center of each cloud and subsequently averaged, resulting in the average cranial shape.

To compare the resulting averaged 3D head shapes (e.g., pre- vs postoperative), they were adjusted in size, tilt, and pitch and aligned to each other using landmarks at the skull base (nasion, bilateral tragions). A heat map scale visualizes areas of relative volume gain and loss.

\section{Statistical Analysis}

Standard statistical software (IBM SPSS Statistics, version 25, IBM Corp.; and GraphPad Prism, version 5.0f, GraphPad) was used for statistical analysis. Because data did not necessarily fit a normal distribution, as assessed by interpretation of z-values for skewness and kurtosis and the Shapiro-Wilk normality test, nonparametric tests were used, and medians and ranges were reported. Parameters of the unpaired 6- and 24-month control cohorts were evaluated with the Mann-Whitney U-test. The pre- and postoperative comparison for the subgroups of operated children was performed using the Wilcoxon matchedpairs signed-rank test. Comparison of the parameters of the 6- and 24-month cohorts with pre- and postoperative data of the endoscopic and conventional cohorts was performed using the Kruskal-Wallis test, followed by a post hoc analysis of the groups using Dunn's multiple comparison test. A p value $<0.05$ was considered statistically significant.

\section{Results \\ Patient Characteristics}

In total, the data of 128 children (48 controls and 80 operated children) with 208 3D photographs were evaluated. There were 30 unaffected children in the 6-month age group and 18 unaffected children in the 24-month age group. The group of endoscopically operated children consisted of 17 with SCS and 16 with MCS, while the group of conventionally operated children included 29 with SCS and 18 with MCS (Table 1). Children undergoing conventional treatment were operated on during the period from January 2014 to December 2018, whereas children undergoing endoscopic operations were operated on from October 2012 to March 2019.

\section{Quantitative Measures}

\section{Sagittal Craniosynostosis}

The results of all measured SCS indices are presented in Table 2 and Fig. 1. The CI was significantly lower at 24 months compared to 6 months, whereas the CCI and the VHI were significantly higher at 24 months, and NFA remained in the same range. The preoperative values of CI and CCI were significantly lower than the 6-month normal control values; only in the conventional group was the preoperative VHI significantly lower than in the 6-month normal control group. Preoperative NFA was no different from the median NFA in 6-month controls. All indices (CI, CCI, VHI, and NFA) were significantly increased postoperatively after both surgical techniques. On comparison of postoperative values of all indices (CI, CCI, VHI, and NFA) to the values of the 24-month normal control cohort, no statistically significant differences were detected. No significant difference between the postoperative values of all indices after endoscopic and conventional correction was demonstrated. 
TABLE 2. Results of SCS

\begin{tabular}{|c|c|c|c|c|c|c|}
\hline \multirow[b]{2}{*}{ SCS } & \multicolumn{2}{|c|}{ Normal Controls } & \multicolumn{2}{|c|}{ Endoscopic } & \multicolumn{2}{|c|}{ Conventional } \\
\hline & 6 Mos & 24 Mos & Preop & Postop & Preop & Postop \\
\hline $\mathrm{Cl}$ & $0.80(0.74-0.93)$ & $0.77(0.73-0.86) \dagger$ & $0.70(0.65-0.82) \ddagger$ & $0.75(0.69-0.86) \dagger$ & $0.69(0.64-0.81) \ddagger$ & $0.73(0.68-0.82) \ddagger$ \\
\hline $\mathrm{CCl}$ & $2.55(2.25-2.72)$ & $2.67(2.23-2.89) \dagger$ & $2.34(2.06-2.54) \ddagger$ & $2.57(2.06-2.77) \ddagger$ & $2.28(2.13-2.58) \ddagger$ & $2.52(2.28-2.76) \ddagger$ \\
\hline $\mathrm{VHI}$ & $1.08(0.89-1.17)$ & $1.13(0.92-1.21)^{\star}$ & $1.04(0.91-1.19) \mathrm{ns}$ & $1.13(0.91-1.26) \dagger$ & $0.99(0.92-1.16) \dagger$ & $1.08(0.97-1.20) \ddagger$ \\
\hline NFA & $122.4(113.4-129.1)$ & $123.6(111.7-133.1) \mathrm{ns}$ & $121.7(102.9-135.5) n s$ & $126.1(115.2-139.5) \ddagger$ & $118.1(104.3-130.1) \mathrm{ns}$ & $124.2(112.8-136.7) \ddagger$ \\
\hline
\end{tabular}

ns = not significant.

Data are given as the median (range). Significant or nonsignificant comparisons in the 24-month and preoperative columns refer to comparisons with values in the 6-month column. Significant or nonsignificant comparisons in the postoperative columns refer to comparisons with values in the preoperative columns. All values in the postoperative columns were nonsignificant compared with those in the 24-month column.

${ }^{*} p<0.05$.

$+p<0.01$.

$\ddagger p<0.001$.

\section{Metopic Craniosynostosis}

All MCS indices are presented in Table 3 and Fig. 2. Briefly, the 24-month values of $30^{\circ} \mathrm{DI}$ and the ECI were significantly lower than the values of the 6-month controls. The preoperative measures of both treatment groups of the CFI and the DI were significantly lower than those of the 6-month controls, whereas only the NFA of the conventional cohort was significantly larger than that of the 6-month controls. The postoperative values of the endoscopic group increased significantly compared to the preoperative values for all 4 indices (CFI, DI, ECI, and NFA). The postoperative values of the conventional group were significantly higher compared to the preoperative state for CFI and DI and significantly lower for ECI; the NFA remained unchanged after conventional correction.

Postoperatively, the ECI and NFA were significantly different from those of the 24-month normal controls only after endoscopic correction. On comparison of endoscopic versus conventional postoperative values, the ECI was significantly higher in the endoscopic group.

\section{Qualitative Results Assessed by Averaged 3D Head Shapes}

In children with SCS, both techniques alter the head shape in a similar pattern, although a differing magnitude between the two techniques can be appreciated (Fig. 3). A relative loss of volume at the forehead and the occiput of the head corresponds with a relative volume gain in the biparietal region, which is more marked after endoscopic correction (Fig. 3A) as compared to the conventional group (Fig. 3B). Comparing the postoperative average 3D head shapes with the 24-month normal controls, the endoscopic group shows relatively more volume in the occipital area (Fig. 3C), while the conventional group displays more volume in the forehead area and occipital area
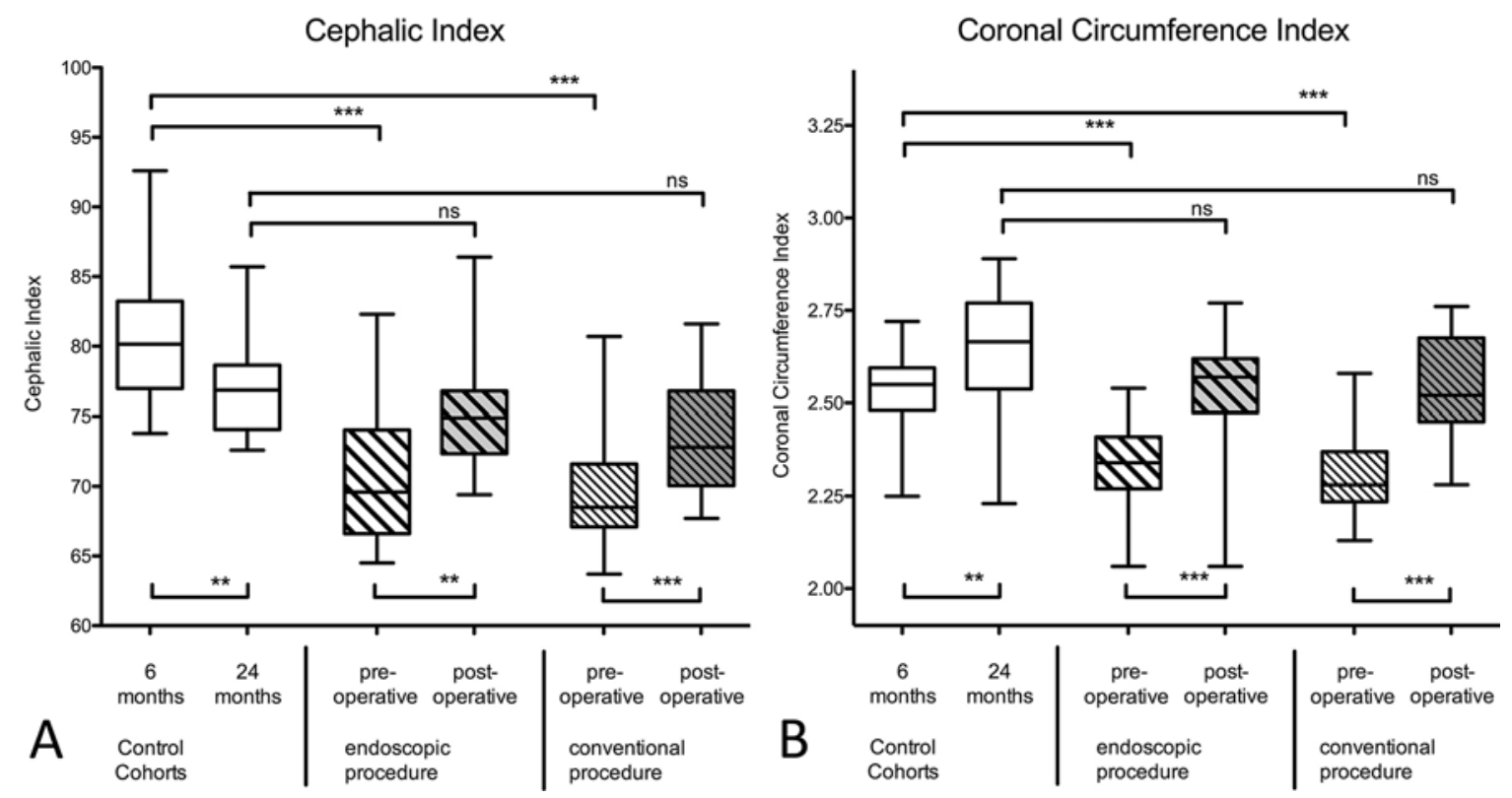

FIG. 1. Sagittal craniosynostosis: comparison of $\mathrm{Cl}$ and $\mathrm{CCl}$ before and after endoscopic and conventional correction and with normal controls at 6 and 24 months of age. ns $=$ not significant. ${ }^{* *} p<0.01,{ }^{* * *} p<0.001$. 
TABLE 3. Results of MCS

\begin{tabular}{|c|c|c|c|c|c|c|}
\hline \multirow[b]{2}{*}{ MCS } & \multicolumn{2}{|c|}{ Normal Controls } & \multicolumn{2}{|c|}{ Endoscopic } & \multicolumn{2}{|c|}{ Conventional } \\
\hline & 6 Mos & 24 Mos & Preop & Postop & Preop & Postop \\
\hline CFI & $1.59(1.56-1.64)$ & $1.59(1.57-1.61) \mathrm{ns}$ & $1.57(1.55-1.62) \ddagger$ & $1.59(1.55-1.63) \dagger$ & $1.56(1.55-1.59) \ddagger$ & $1.58(1.57-1.64) \ddagger$ \\
\hline $30^{\circ} \mathrm{DI}$ & $0.96(0.94-0.98)$ & $0.93(0.91-0.96) \ddagger$ & $0.92(0.89-0.96) \ddagger$ & $0.95(0.91-0.99) \dagger$ & $0.92(0.88-0.94) \ddagger$ & $0.93(0.90-0.96) \dagger$ \\
\hline $\mathrm{ECl}$ & $0.79(0.75-0.87)$ & $0.77(0.73-0.89) \dagger$ & $0.80(0.72-0.90) \mathrm{ns}$ & $\begin{array}{l}0.82(0.78-0.90)^{*} \\
\text { (vs } 24-m o \text { group) } \ddagger\end{array}$ & $0.79(0.74-0.84)$ & $\begin{array}{c}0.76(0.72-0.84)^{*} \\
\text { (vs postop endoscopic } \\
\text { group) } \ddagger\end{array}$ \\
\hline NFA & $122.4(113.4-129.1)$ & $123.6(111.7-133.1) \mathrm{ns}$ & $125.7(114.8-136.2) n s$ & $\begin{array}{c}132.8 \text { (121.6-140.7) } \\
\text { (vs 24-mo group) } \ddagger\end{array}$ & $127.1(106.2-143.8)^{*}$ & $127.5(112.5-139.4) n s$ \\
\hline
\end{tabular}

Data are given as median (range). Significant or nonsignificant comparisons in the 24-month and preoperative columns refer to comparisons with values in the 6-month column. Significant or nonsignificant comparisons in the postoperative columns refer to comparisons with values in the preoperative columns. All values in the postoperative columns were nonsignificant compared with those in the 24-month column, except in the endoscopic postoperative group for ECI and NFA.

${ }^{*} p<0.05$.

$\dagger p<0.01$.

$\ddagger p<0.001$.

(Fig. 3D). There is still less volume in the parietal area of both groups, which is more noticeable in the conventional group (Fig. 3D). Overall, the endoscopic group matches more homogeneously with the 24-month controls, as indicated by a more uniform distribution of smaller deviations over a wider frontoparietal area.

For MCS, the techniques appear to alter the head shape slightly differently (Fig. 4). Both techniques increased the volume of the forehead bilaterally and decreased the volume of the biparietal region. However, after conventional correction, the relative volume gain projected more frontally and laterally in the forehead area above the lateral orbits (Fig. 4B), while after endoscopic correction the relative volume gain was distributed from the bilateral forehead area-sparing the midportion of the forehead-to a maximum at the bregma, reaching the vertex area posteriorly (Fig. 4A). On comparison with 24-month controls, the conventional group shows relatively less volume at the curvatures of the lateral forehead immediately above the orbits, whereas the endoscopic group displays relatively less volume across the whole forehead area and more volume centered at the vertex area (Fig. 4C and D). Overall, the average 3D mesh after conventional correction more closely matches the average 24-month mesh, as indicated by a more uniform distribution of less marked deviations.

\section{Discussion}

The presented study investigated quantitative and qualitative changes of head shape after endoscopic and conventional correction of SCS and MCS, and in comparison
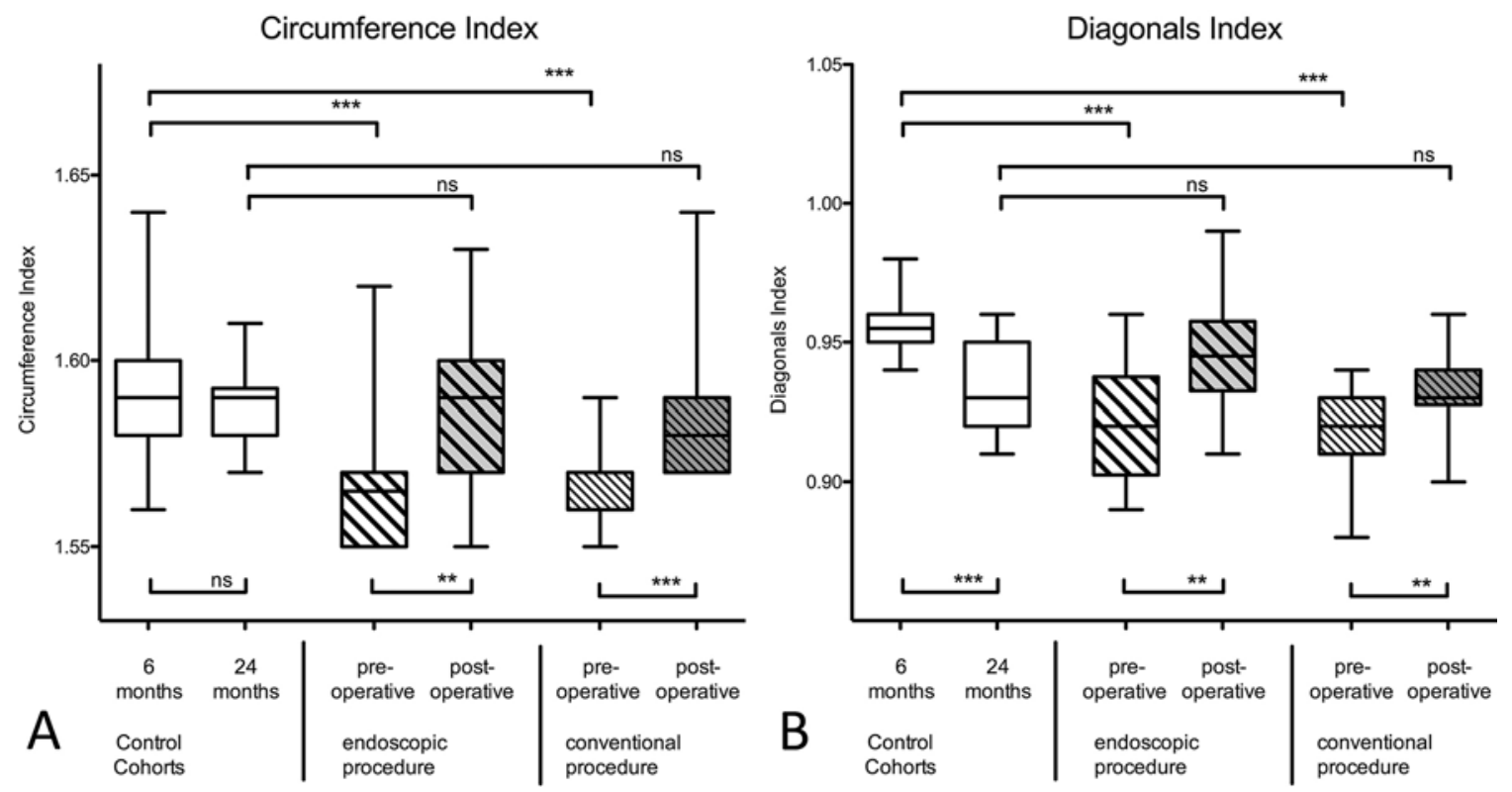

FIG. 2. Metopic craniosynostosis: comparison of $\mathrm{Cl}$ and $\mathrm{DI}$ before and after endoscopic and conventional correction and with normal controls at 6 and 24 months of age. ${ }^{* *} p<0.01,{ }^{* *} p<0.001$. 

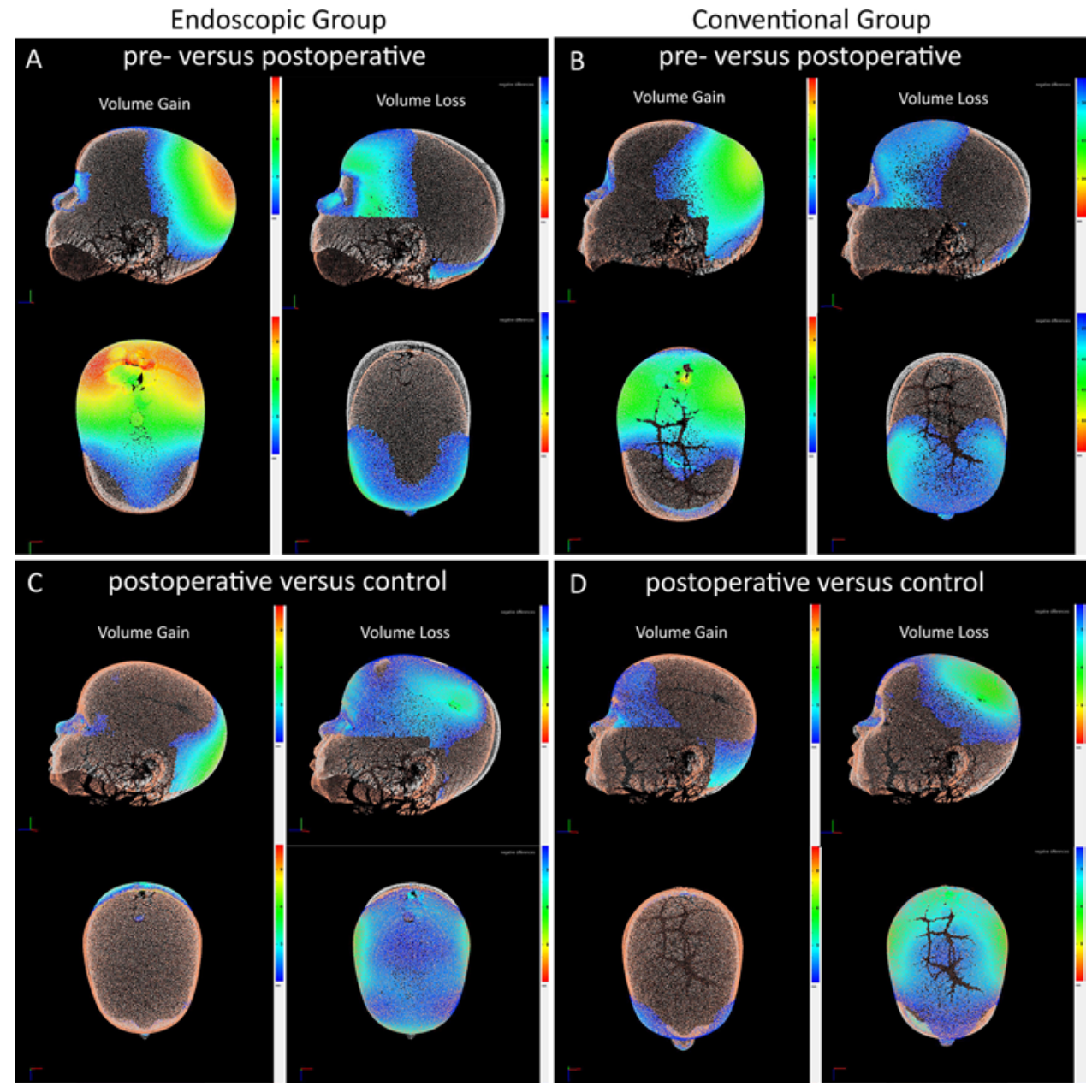

FIG. 3. Relative changes shown as heat maps after endoscopic and conventional correction for SCS. The upper row shows the pre- versus postoperative superimposition of the respective average 3D head shape for endoscopic $(\mathbf{A})$ and conventional $(\mathbf{B})$ correction. In the lower row, the superimposition of average 3D head shapes of postoperative versus 24-month normal controls for endoscopic (C) and conventional (D) correction demonstrates areas of less and more volume in comparison to the controls.

with age-matched controls. Both the analysis of quantitative measurements and the qualitative superimposition of averaged 3D head shapes demonstrated a similar evolution toward the average head shape of the control group after both endoscopic and conventional correction, but also revealed differing patterns. The qualitative evaluation depicted a closer match of the average head shape with the control group after endoscopic correction for SCS and after conventional correction for MCS.

The presentation of an infant with craniosynostosis constitutes a situation in which a potentially extensive operative procedure is discussed with the parents, which has immediate and long-standing implications on several aspects of the child's future life. The multiplicity of operative techniques and timed approaches arises from the absence of solid (prospective and multicenter) data comparing those techniques and considering all relevant outcome parameters.

Several variables potentially qualify to assess outcome after craniosynostosis correction, such as efficacy and safety of the procedure, as reflected by data on the surgi- cal intervention and the neurocognitive and aesthetic outcome. Most comparisons have focused on variables of the surgical intervention such as operation time, blood loss, hospital stay, and complication rates, among others, and a multiplicity of publications reported on those, with most data favoring an endoscopic approach. ${ }^{14-18}$ The impact of craniosynostosis correction and timing on neurocognitive outcome is an area of ongoing debate and, despite its indisputable importance, is beyond the scope of this paper. ${ }^{19-23}$ To evaluate aesthetic outcome, there is a subjective perspective of the patients, parents, and surgeon, and an objective perspective by morphometric measurements. Three-dimensional photography as an alternative to radiation-based techniques is a well-established method, which reliably allows assessment of volumetric and linear dimensions. ${ }^{24-29}$ To avoid unnecessary radiation exposure and its inherit risks, ${ }^{30,31}$ all radiation-based investigations to assess nonsyndromic children, such as CT or radiography, were abandoned in our center more than a decade ago.

Because individual head shape is a complex 3D model 

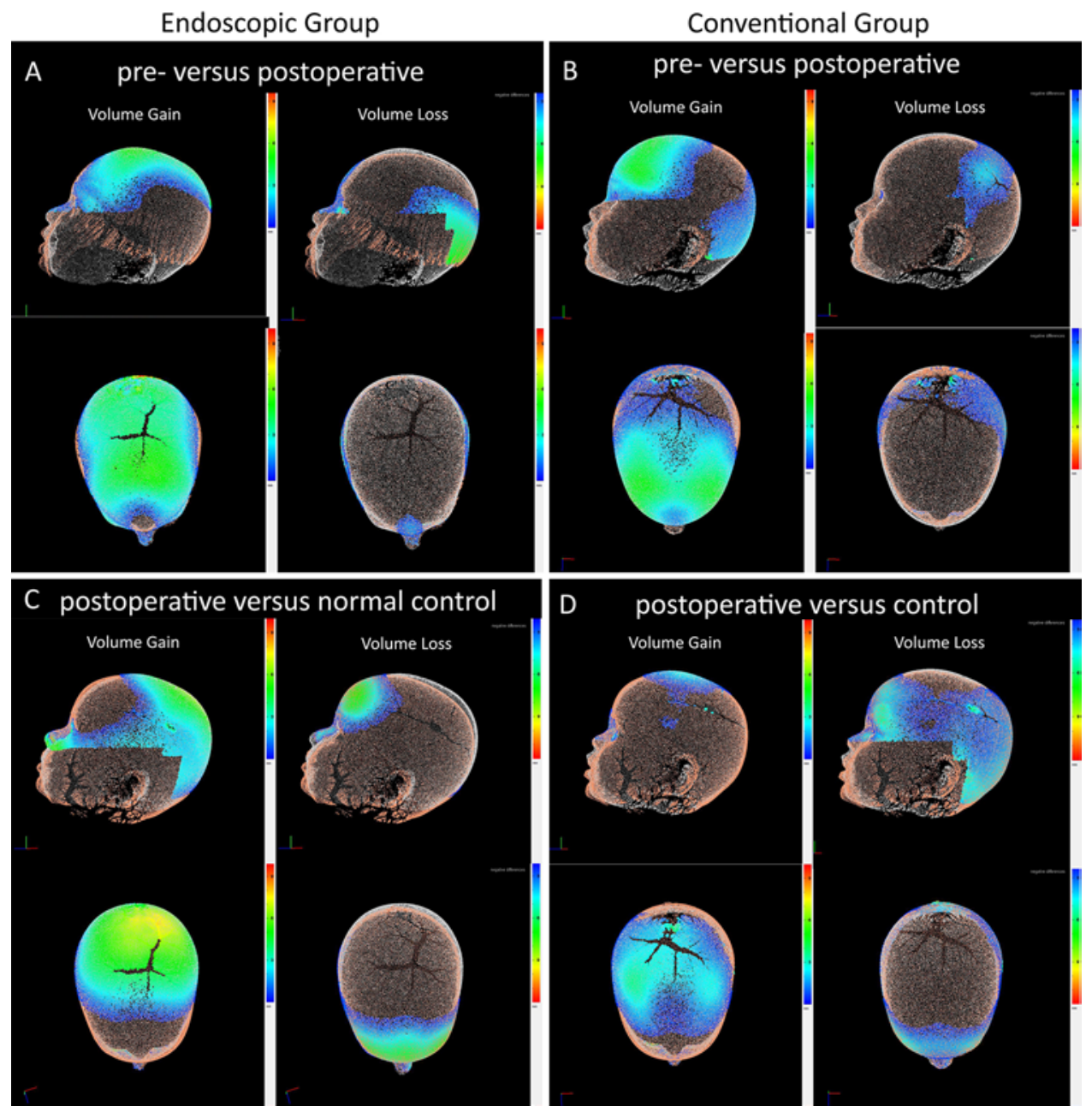

FIG. 4. Relative changes shown as heat maps after endoscopic and conventional correction for MCS. The upper row shows the pre- versus postoperative superimposition of the respective average 3D head shape for endoscopic (A) and conventional (B) correction. In the lower row, the superimposition of average 3D head shapes of postoperative versus 24-month normal controls for endoscopic (C) and conventional (D) correction demonstrates areas of less and more volume in comparison to the controls.

with a wide variation among a population and across an individual lifespan, no single linear parameter will sufficiently express the alteration induced by a craniosynostosis or any surgical correction. As an approximation, a combination of indices describing aspects of the altered craniosynostotic head shape was used. The utilization of indices, and not absolute measures, eliminates the influence of physiological head growth and allows comparison. For SCS, the CI to depict increased anterior-posterior dimension in relation to width, the CCI to depict the dimension of the biparietal contour in the coronal plane, and the VHI as a surrogate of vertex height were used to describe aspects of the altered cranial shape in SCS. For MCS, the axial CFI was utilized to depict alternation of shape by forward positioning of the bilateral forehead, the $30^{\circ} \mathrm{DI}$ toward the lateral forehead to describe forward positioning in this bilateral dimension, and the ECI to describe the position of the bilateral exocanthions in relation to a midpoint of the forehead. For both SCS and MCS, NFA characterizes the inclination of the forehead.

\section{Outcomes After SCS and MCS}

Both surgical strategies for SCS resulted in a significant increase of the CI and CCI compared to matched preoperative values. Intergroup comparison indicated that both techniques modified those indices: whereas preoperatively they significantly differed from those of the 6-month normal controls, the respective postoperative values were nonsignificantly different from the values of 24-month controls. The median of 0.73 after conventional correction was slightly below the value of normocephaly. Although the VHI increased significantly after both surgical techniques, a similar difference was shown between the 6and 24-month cohorts. A possible effect of treatment on vertex height was demonstrated only for the conventional technique. In contrast, although the NFA did not change in the natural course from 6 to 24 months and increased significantly from pre- to postoperatively after both types of correction, the intergroup comparison did not detect significant differences between the groups. The qualitative 
evaluation by superimposed pre- and postoperative average 3D head shapes confirmed these findings: both techniques resulted in a relative shortening of the head accompanied by volume gain of the parietal region. While the quantitative measurements did not show any significant difference between endoscopic and conventional groups, the qualitative evaluation did. The relative volume gain in the parietal area was more pronounced in the endoscopic group. This resulted in a better matched overlay and fewer deviations from the 24-month normal controls, and indicates an overall closer approximation to the normal population after endoscopic correction of SCS as compared to the applied conventional correction technique. Apart from the performed surgical technique, two factors influenced the achieved result and possibly account for the demonstrated differing outcome: younger age at surgery and the utilization of helmet therapy after endoscopic SCS correction. In consideration of the efficacy of helmet therapy alone to alter head shapes even prior to surgical correction of SCS, ${ }^{32}$ a significant contribution of helmet therapy to the demonstrated difference between the two techniques is likely. Similar results after an earlier conventional procedure with subsequent helmet therapy might be achievable.

In MCS, the development of the examined indices differed between both surgical techniques, unlike in SCS, in which all examined indices changed in the same direction. Alteration of head shape, such as increased diagonal dimensions toward the lateral curvature of the forehead, were achieved by both surgical techniques and could be confirmed by pre- and postoperative assessment of the CFI and DI. In contrast, the ECI increased after endoscopic correction, whereas it decreased after conventional correction and in the 24-month cohort, resulting in a significant difference. Correspondingly, the NFA increased significantly after endoscopic correction, resulting in a significantly larger angle as compared to 24-month controls. This implies that the midpoint of the forehead in the endoscopic group is positioned more posterior in relation to the exocanthions (ECI), indicating a more posteriorly tilted (NFA) and positioned central part of the forehead. This is confirmed by the qualitative analysis demonstrating relatively less volume in this area. After endoscopic correction, the relative volume gain not only involves the desired area of the lateral forehead, but also involves a midline volume gain from bregma to vertex. These changes are not induced by the strip craniectomy alone, but appear to represent the compensatory growth direction secondary to the helmet therapy. In contrast to the potential of helmet therapy to substantially alter head shape after SCS correction, the influence after MCS correction is less pronounced and requires a longer duration of helmet therapy due to the involvement of the anterior skull base in MCS, a spatially more connected and fixed osseous structure compared to the moldable calvarial shape above the skull base in SCS. After the conventional technique, the depicted area with maximum volume gain corresponds well to the site of an FOR procedure. Overall, the match to the 24-month controls appears more homogeneously after conventional correction.

\section{Comparison of Techniques}

In summary, we could demonstrate that both surgical techniques for SCS and MCS were capable of positively altering the cranial shape toward that of normal controls, but qualitatively superior results were noted after endoscopic SCS correction with subsequent helmet therapy and after conventional MCS correction. As initially discussed, the morphometric, aesthetic aspect is only one among others to be considered. The presented data do not evaluate the subjective perception of the morphometric result as experienced by the parents and the child, which ultimately might be the most important outcome parameter. Considering the obvious differences of endoscopic and conventional techniques with regard to other parameters such as invasiveness, hospital stay, rate of blood transfusion, and necessary helmet therapy, we are reluctant to draw conclusions about the superiority of one technique based only on morphometric outcomes. Nevertheless, the presented data are of high value for a balanced discussion when counseling families about possible treatment options.

\section{Limitations}

Despite the relatively large overall number of 128 children with 3D photography scans, the individual group size remains rather small with a predominance of male children. While the conventional techniques were standard and routinely used during the period of this study, the data of endoscopic procedures incorporate a learning curve. This is reflected by factors such as a decreasing duration of helmet therapy and adaptations of the surgical technique for endoscopic MCS repair reflecting the knowledge gained to sufficiently reach the nasofrontal suture to allow optimal correction.

The utilization of a limited number of indices can only approximately describe the complex and changing cranial 3D head shape, but nevertheless will allow an objective comparison. Obviously, a subjective aspect is involved in the qualitative assessment of the superimposed, averaged $3 \mathrm{D}$ head shapes.

\section{Conclusions}

The presented data confirm that endoscopic and conventional correction of SCS and MCS alter the head shape toward those of a control group. Average 3D head shapes acquired after endoscopic SCS and conventional MCS correction matched more homogeneously with the average 3D head shape of controls after 24 months. While this study provides robust data on morphometric outcomes after craniosynostosis correction, only an assessment of the entire multiplicity of outcome parameters will allow valid conclusions of superiority of one surgical technique. Hence, further, preferably multicenter, studies are needed to collect data on more aspects, including subjective patient assessment of the achieved result as a relevant outcome parameter.

\section{References}

1. Cornelissen M, den Ottelander B, Rizopoulos D, et al. Increase of prevalence of craniosynostosis. J Craniomaxillofac Surg. 2016;44(9):1273-1279.

2. Ridgway EB, Berry-Candelario J, Grondin RT, et al. The management of sagittal synostosis using endoscopic suturec- 
tomy and postoperative helmet therapy. J Neurosurg Pediatr. 2011;7(6):620-626.

3. Riordan CP, Zurakowski D, Meier PM, et al. Minimally invasive endoscopic surgery for infantile craniosynostosis: a longitudinal cohort study. J Pediatr. 2020;216:142-149.e2.

4. Jimenez DF, Barone CM. Endoscopic craniectomy for early surgical correction of sagittal craniosynostosis. J Neurosurg. 1998;88(1):77-81.

5. Jimenez DF, Barone CM, Cartwright CC, Baker L. Early management of craniosynostosis using endoscopic-assisted strip craniectomies and cranial orthotic molding therapy. Pediatrics. 2002;110(1 Pt 1):97-104.

6. Isaac KV, MacKinnon S, Dagi LR, et al. Nonsyndromic unilateral coronal synostosis: a comparison of fronto-orbital advancement and endoscopic suturectomy. Plast Reconstr Surg. 2019;143(3):838-848.

7. Isaac KV, Meara JG, Proctor MR. Analysis of clinical outcomes for treatment of sagittal craniosynostosis: a comparison of endoscopic suturectomy and cranial vault remodeling. J Neurosurg Pediatr. 2018;22(5):467-474.

8. Jimenez DF, McGinity MJ, Barone CM. Endoscopy-assisted early correction of single-suture metopic craniosynostosis: a 19-year experience. J Neurosurg Pediatr. 2018;23(1):61-74.

9. Magge SN, Bartolozzi AR, Almeida ND, et al. A comparison of endoscopic strip craniectomy and pi craniectomy for treatment of sagittal craniosynostosis. J Neurosurg Pediatr. 2019; 23(6):708-714.

10. Porras AR, Tu L, Tsering D, et al. Quantification of head shape from three-dimensional photography for presurgical and postsurgical evaluation of craniosynostosis. Plast Reconstr Surg. 2019;144(6):1051e-1060e.

11. Meulstee JW, de Jong GA, Borstlap WA, et al. The normal evolution of the cranium in three dimensions. Int J Oral Maxillofac Surg. 2020;49(6):739-749.

12. Mercan E, Hopper RA, Maga AM. Cranial growth in isolated sagittal craniosynostosis compared with normal growth in the first 6 months of age. J Anat. 2020;236(1):105-116.

13. Katanacho M, Lack JC, Fehlhaber F, Krüger J. Registration of pre- and postoperative surface scans for pediatric neurosurgery. Curr Dir Biomed Eng. 2018;4(1):657-660.

14. Yan H, Abel TJ, Alotaibi NM, et al. A systematic review and meta-analysis of endoscopic versus open treatment of craniosynostosis. Part 1: the sagittal suture. J Neurosurg Pediatr. 2018;22(4):352-360.

15. Yan H, Abel TJ, Alotaibi NM, et al. A systematic review of endoscopic versus open treatment of craniosynostosis. Part 2: the nonsagittal single sutures. J Neurosurg Pediatr. 2018; 22(4):361-368

16. Thompson DR, Zurakowski D, Haberkern CM, et al. Endoscopic versus open repair for craniosynostosis in infants using propensity score matching to compare outcomes: a multicenter study from the Pediatric Craniofacial Collaborative Group. Anesth Analg. 2018;126(3):968-975.

17. Goyal A, Lu VM, Yolcu YU, et al. Endoscopic versus open approach in craniosynostosis repair: a systematic review and meta-analysis of perioperative outcomes. Childs Nerv Syst. 2018;34(9):1627-1637.

18. Rattani A, Riordan CP, Meara JG, Proctor MR. Comparative analysis of cranial vault remodeling versus endoscopic suturectomy in the treatment of unilateral lambdoid craniosynostosis. J Neurosurg Pediatr. 2020;26(2):105-112.

19. Kljajić M, Maltese G, Tarnow P, et al. The cognitive profile of children with nonsyndromic craniosynostosis. Plast Reconstr Surg. 2019;143(5):1037e-1052e.

20. Knight SJ, Anderson VA, Spencer-Smith MM, Da Costa AC. Neurodevelopmental outcomes in infants and children with single-suture craniosynostosis: a systematic review. $\mathrm{Dev} \mathrm{Neu-}$ ropsychol. 2014;39(3):159-186.
21. Mandela R, Bellew M, Chumas P, Nash H. Impact of surgery timing for craniosynostosis on neurodevelopmental outcomes: a systematic review. J Neurosurg Pediatr. 2019;23(4): 442-454.

22. Osborn AJ, Roberts RM, Mathias JL, et al. Cognitive, behavioral and psychological functioning in children with metopic synostosis: a meta-analysis examining the impact of surgical status. Child Neuropsychol. 2019;25(2):263-277.

23. Patel A, Yang JF, Hashim PW, et al. The impact of age at surgery on long-term neuropsychological outcomes in sagittal craniosynostosis. Plast Reconstr Surg. 2014;134(4):608e617 e.

24. Brons S, van Beusichem ME, Bronkhorst EM, et al. Methods to quantify soft tissue-based cranial growth and treatment outcomes in children: a systematic review. PLoS One. 2014; 9(2):e89602.

25. de Jong G, Tolhuisen M, Meulstee J, et al. Radiation-free 3D head shape and volume evaluation after endoscopically assisted strip craniectomy followed by helmet therapy for trigonocephaly. J Craniomaxillofac Surg. 2017;45(5):661-671.

26. Linz C, Meyer-Marcotty P, Böhm H, et al. 3D stereophotogrammetric analysis of operative effects after broad median craniectomy in premature sagittal craniosynostosis. Childs Nerv Syst. 2014;30(2):313-318.

27. Schaaf H, Pons-Kuehnemann J, Malik CY, et al. Accuracy of three-dimensional photogrammetric images in non-synostotic cranial deformities. Neuropediatrics. 2010;41(1):24-29.

28. McKay DR, Davidge KM, Williams SK, et al. Measuring cranial vault volume with three-dimensional photography: a method of measurement comparable to the gold standard. $J$ Craniofac Surg. 2010;21(5):1419-1422.

29. Katina S, McNeil K, Ayoub A, et al. The definitions of threedimensional landmarks on the human face: an interdisciplinary view. J Anat. 2016;228(3):355-365.

30. Pearce MS, Salotti JA, Little MP, et al. Radiation exposure from CT scans in childhood and subsequent risk of leukaemia and brain tumours: a retrospective cohort study. Lancet. 2012;380(9840):499-505.

31. Miglioretti DL, Johnson E, Williams A, et al. The use of computed tomography in pediatrics and the associated radiation exposure and estimated cancer risk. JAMA Pediatr. 2013; 167(8):700-707.

32. Hashmi A, Marupudi NI, Sood S, Rozzelle A. Effect of preoperative molding helmet in patients with sagittal synostosis. J Craniofac Surg. 2017;28(4):898-903.

\section{Disclosures}

Christoph Blecher is the founder of Cranioform, who is responsible for the planning and manufacturing of the head orthosis after endoscopic procedures.

\section{Author Contributions}

Conception and design: Schulz, Paulikat, Thomale. Acquisition of data: Schulz, Liebe-Püschel, Seelbach, Fehlhaber, Schwarz, Blecher, Thomale. Analysis and interpretation of data: all authors. Drafting the article: Schulz, Paulikat, Fehlhaber, Blecher. Critically revising the article: Schulz, Liebe-Püschel, Seelbach, Fehlhaber, Schwarz, Blecher, Thomale. Reviewed submitted version of manuscript: Schulz, Thomale. Approved the final version of the manuscript on behalf of all authors: Schulz. Statistical analysis: Schulz, Thomale.

\section{Correspondence}

Matthias Schulz: Charité Universitätsmedizin Berlin, Germany. matthias.schulz@charite.de. 